\title{
Two Cases of Cerebral
}

\section{Involvement in Malignant}

Lymphoma (CD20+) That Responded to Combination Therapy with Rituximab and Cladribine

\author{
Tatsuro Jo Masatoshi Matsuo Kensuke Horio \\ Masao Tomonaga \\ Department of Hematology, Japanese Red Cross Society Nagasaki Genbaku \\ Hospital, Nagasaki, Japan
}

\section{Key Words}

Cladribine $\cdot$ Rituximab $\cdot$ Cerebral involvement in malignant lymphoma .

Purine analogue

\begin{abstract}
Cerebral involvement frequently occurs in association with progression or relapse of malignant lymphoma. Chemotherapy with cyclophosphamide, doxorubicin, vincristine, and prednisolone, the standard chemotherapy for malignant lymphoma, is an ineffective treatment for cerebral involvement because these drugs cannot cross the blood-brain barrier. Therefore, various alternative strategies have been attempted. Although high-dose methotrexate combined with whole-brain radiotherapy is widely used to treat primary central nervous system lymphoma, there is no standard therapy to treat cerebral involvement in malignant lymphoma. Furthermore, high-dose methotrexate in combination with whole-brain radiotherapy is not always effective, and high rates of neurotoxicity are often observed, particularly in the elderly. To expand the therapeutic options for central nervous system involvement in recent years, systemic chemotherapies, including rituximab, high-dose methotrexate, and other agents that act during the S, G2, and M phases of the cell cycle, have been attempted. In our hospital, cladribine, a purine analogue with a cytocidal effect on resting malignant cells (G0/G1 phase of the cell cycle), has been used in combination with rituximab, which exhibits antitumor effects on nodal and extranodal lesions of relapsed and/or refractory B cell lymphomas, particularly cerebral lesions. Here, we report 2 representative cases of patients who were treated with cladribine plus rituximab and survived for 30 months (died of sepsis) and 52 months (still alive), respectively. The
\end{abstract}


outcomes of these cases suggest that cladribine plus rituximab combination therapy with whole-brain radiotherapy may be very useful as salvage therapy for secondary central nervous system lymphoma and as initial therapy for primary central nervous system lymphoma.

\section{Introduction}

Cyclophosphamide, doxorubicin, vincristine, and prednisolone (CHOP) therapy is an effective systemic chemotherapy for non-Hodgkin's lymphoma, and CHOP-based regimens have been widely used. However, most of these drugs cannot cross the bloodbrain barrier, and thus they fail to attain effective concentrations in the brain. Indeed, many researchers have reported that CHOP therapy is not very effective for primary central nervous system lymphoma (CNSL) or malignant lymphoma with cerebral or meningeal involvement.

Various other therapeutic strategies for primary CNSL have been attempted. Of note, systemic high-dose methotrexate (MTX; $\geq 3 \mathrm{~g} / \mathrm{m}^{2}$ ) combined with whole-brain radiotherapy (WBRT; 30-45 Gy) was shown to be very effective in prolonging survival time, and still plays a central role in the treatment of primary CNSL. However, this therapy is associated with serious side effects, including renal dysfunction, hepatic dysfunction, interstitial pneumonia, and myelosuppression, as well as leukoencephalopathy in elderly patients. A new therapeutic option for both nodal and extranodal lesions is rituximab, a chimeric monoclonal antibody against the CD20 antigen. Rituximab alone has limited efficacy, but in combination with other drugs (e.g. temozolomide) its usefulness after progression or relapse of primary CNSL has been suggested.

In our department, rituximab plus cladribine (RC) combination therapy has been used for relapsed and/or refractory B cell lymphomas. Cladribine (2-chloro-2'deoxyadenosine) is a purine analogue with a very potent inhibitory effect on the proliferation of lymphoma. In cells, cladribine is phosphorylated to the pharmacologically active form 2-CdATP, and then incorporated into the DNA chain of both proliferating and resting cells, which causes cell death. The presence of 2-CdAmetabolizing enzyme (adenosine deaminase) in lymphocytes and monocytes impedes the increase in the intracellular concentration of active 2-CdATP. By substituting chlorine for hydrogen in deoxyadenosine, cladribine is protected from enzymatic degradation.

Treatment of relapsed or refractory non-Hodgkin's lymphoma, unsuccessfully treated with CHOP therapy, will need to be effective for both nodal and extranodal lesions and to have no cross-resistance to CHOP therapy. RC combination therapy fulfills these requirements. In this article, we report 2 cases of malignant B-cell lymphoma with cerebral involvement responsive to RC combination therapy. 


\section{Case Presentations}

\section{Case 1}

High lactate dehydrogenase was found in a 76-year-old man by a neighborhood physician at the end of 1999; however, the cause was not investigated. In March 2003, the patient visited our department with a chief complaint of general malaise. Abdominal CT revealed splenomegaly, and acute exacerbation of indolent malignant lymphoma was suspected. In April 2003, he was admitted to our department for close examination and treatment. Although no superficial lymph node swelling was found upon admission, whole-body CT revealed some enlargement of the submandibular-cervical and mediastinal lymph nodes, and ${ }^{67} \mathrm{Ga}$ scintigraphy revealed intense ${ }^{67} \mathrm{Ga}$ uptake by the spleen. Furthermore, a stomach biopsy specimen contained malignant lymphoma cells (CD20+ B cells), and the bone marrow aspirate contained abnormal lymphocytes.

Also in April 2003, the patient underwent total gastrectomy, splenectomy, and cholecystectomy, and was diagnosed with stage IV intravascular large B-cell lymphoma (CD5-, CD10-, CD20+, CD45RO-, bcl2+, bcl6-, EBER(ISH)-). After 4 cycles of rituximab, pirarubicin, cyclophosphamide, vincristine, and prednisolone (R-THP-COP) therapy, the nodal lesions were generally reduced, which led to the patient's discharge in July 2003. He was given 2 additional cycles of R-THP-COP; however, in September 2003, he required rehospitalization after a brain CT revealed many central nervous system lesions. High-dose MTX combined with WBRT (total dose of $36 \mathrm{~Gy}$ ) was immediately administered, but in vain. Given his advanced age and weakness, high-dose cytosine arabinoside therapy was not considered. Instead, he received 2 cycles of RC combination therapy $\left(375 \mathrm{mg} / \mathrm{m}^{2}\right.$ rituximab intravenously on day 1 and $0.1 \mathrm{mg} / \mathrm{kg}$ cladribine intravenously once a day on days 2-6), and most of his intracranial lesions disappeared by November 2003. He was ambulatory on discharge and had no neurological symptoms. He completed another 4 cycles of RC combination therapy by April 2004 (fig. 1), and complete response (Response Evaluation Criteria in Solid Tumors (RECIST) classification) was maintained until March 2006. After 6 cycles of RC combination therapy, pancytopenia was observed in May 2004. Chemotherapy-related myelodysplastic syndrome (MDS) was suspected by laboratory examination, including bone marrow aspiration. In January 2006, he developed a high fever and was urgently admitted to our department where he was treated for recurrent Gram-positive and Gramnegative sepsis. In March 2006, he was transferred to another hospital for hospice care because he wished no further aggressive treatment. No neurotoxicity due to chemotherapy and WBRT was observed during the entire course of treatment.

Case 2

A 64-year-old man consulted a neighborhood physician in January 2008 because of memory loss and disorientation, which had become severe at the end of 2007. Brain MRI revealed a neoplastic lesion in the left splenium of the corpus callosum and in the medial side of the trigone of the left lateral ventricle and an extensive perilesional high-intensity area that appeared to be white matter edema ( $\underline{\text { fig. 2) }}$. At the end of January 2008, he was admitted to our department for close examination and treatment.

Splenic lymphoma with villous lymphocytes with central nervous system involvement and bone marrow invasion by malignant lymphoma cells (B cell, CD20+) was diagnosed based on whole-body CT, brain MRI, lumbar puncture, and bone marrow aspiration. RC combination therapy with WBRT (40 Gy) was started, and his clinical symptoms immediately decreased after 1 cycle (before the end of WBRT). High-dose MTX therapy was not utilized because the lymphoma cells had spread to various tissues, including the brain, and an adequate effect could not be expected with MTX therapy alone.

In June 2008, after 4 cycles ( 1 cycle every 4 weeks) of RC combination therapy, the intracranial lesion was markedly reduced, and the surrounding white matter edema improved (fig. 2). The treatment response was considered a complete response, and this status has been maintained for more than 52 months. To avoid myelosuppression, RC combination therapy was finished in 4 cycles. However, the patient unfortunately developed leukoencephalopathy in January 2010. 


\section{Discussion}

Primary CNSL is a rare type of brain tumor and a rare form of malignant lymphoma. However, cerebral involvement frequently occurs in association with progression or relapse of malignant lymphoma. CHOP therapy, the standard chemotherapy for malignant lymphoma, is an inefficient treatment for cerebral involvement because of the blood-brain barrier. Therefore, various alternative strategies have been attempted. Since surgical removal of CNSL alone results in a mean survival of 1-2 months [1], it is not performed in patients with an established diagnosis of malignant lymphoma. In addition, steroids are known to reduce CNSL, but usually only for a few months. Until recently, WBRT played a central role in the treatment of primary CNSL. However, Nelson et al. [2] reported a relapse rate of over $80 \%$ within the whole-brain irradiation field and boost irradiation field. In addition, the 5-year survival rate was as low as 7\% $[3,4]$, indicating that primary CNSL may be difficult to control with WBRT alone. The results of many subsequent studies have led to the adoption of high-dose MTX combined with WBRT as a current mainstay of primary CNSL treatment. High-dose MTX therapy for primary CNSL began as high-dose MTX with leucovorin rescue, which was first attempted in the late 1970s. It was subsequently combined with WBRT, which improved prognosis (median survival: 33-39 months) [5, 6].

Rituximab is under vigorous study as a therapeutic option for patients with progression or relapse despite initial or second-line therapy with high-dose MTX plus WBRT. Rituximab, which was established as a part of R-CHOP therapy, is also expected to be a useful therapeutic option for primary CNSL because it enters the cerebrospinal fluid after intravenous administration [7]. However, a series of studies reported that rituximab monotherapy had limited efficacy, and consequently combination therapy had to be considered. Therefore, we have focused on RC, which is approved for the treatment of non-Hodgkin's lymphoma. Cladribine, a purine analogue, inhibits DNA synthesis and may induce apoptosis during the proliferative and quiescent phases of the cell cycle by depleting nicotinamide adenine dinucleotide and adenosine triphosphate.

We originally introduced RC combination therapy as a salvage treatment for refractory aggressive and indolent lymphoma. Subsequently, we found that this combination is more effective for CNSL, as shown by the 2 cases described above. Delgado et al. [8] reported that cladribine entered the cerebrospinal fluid, and Fabianowska-Majewska et al. [9] demonstrated the potent cytocidal effect of cladribine on human primary CNSL cells in vitro and discussed potential mechanisms. RC combination therapy was administered after high-dose MTX combined with WBRT, a conventional therapy for primary CNSL, and complete response was obtained in case 1. Furthermore, 1 cycle of RC combination therapy began to exhibit an antitumor effect even before the completion of WBRT in case 2 . These 2 cases suggest that cladribine could cross the blood-brain barrier and reach sufficient cerebrospinal fluid levels to have an antitumor effect not only in vitro but also in lymphoma patients. Cladribine has potent effects in both proliferating and resting lymphoma cells and has less nonhematological adverse events such as hair loss, renal dysfunction, hepatic dysfunction, interstitial pneumonia, and cardiac toxicity than other anticancer drugs that act during the post-G1 phase of the cell cycle. Therefore, cladribine is a useful choice as salvage chemotherapy for relapsed and/or refractory lymphomas. However, with the use of 
cladribine, we have to pay attention to myelosuppression. Secondary MDS arose in case 1. He was pretreated with 6 cycles of R-THP-COP therapy and high-dose MTX combined with WBRT. Therefore, some bone marrow damage might have existed prior to RC combination therapy. In addition, considering myelosuppression, the possibility that 6 cycles of RC combination therapy was excessive is undeniable. The cytotoxic effect during the G0/G1 phase of the cell cycle may harm hematopoietic stem cells and may be connected with MDS. In case 2, 4 cycles of RC combination therapy was sufficient to obtain an antitumor effect, and secondary MDS did not occur. For the above reason, we now only administer up to 4 cycles of RC combination therapy.

The risk of WBRT-related neurotoxicity is unacceptably high. Recent studies have focused on either chemotherapy alone or reduced-dose WBRT, but the problem of WBRT dose remains unsettled [10]. The 2 cases presented in this paper suggest the possibility that the combination of WBRT with RC combination therapy may be able to reduce the dose of WBRT, and thus reduce the risk of WBRT-related neurotoxicity.

In summary, many studies, as well as our own experience, indicate that RC combination therapy may be very useful as initial therapy for primary CNSL and as salvage therapy for secondary CNSL, pending future evaluations of its efficacy, safety, and effect on life expectancy. Our 2 patients with cerebral involvement in malignant lymphoma (CD20+) achieved complete response with survival times of 30 months (case 1, patient died due to sepsis) and 52 months (case 2, patient is still alive) in response to treatment with RC combination therapy. Although RC combination therapy is potentially effective against progression or relapse of previously treated CNSL, the therapy may need to be stopped if the bone marrow is suppressed and the disease progresses, and measures may need to be taken to control susceptibility to infection. 
11 Sep 2003

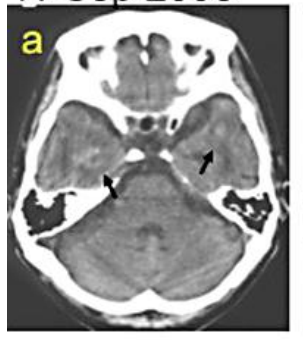

14 May 2004

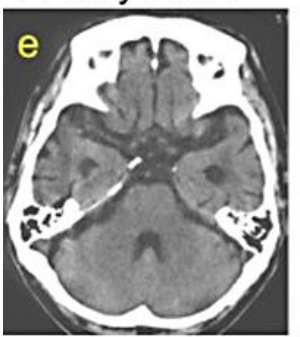

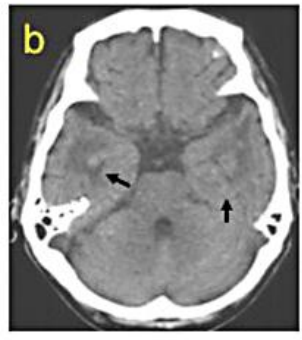

Pre-RC therapy
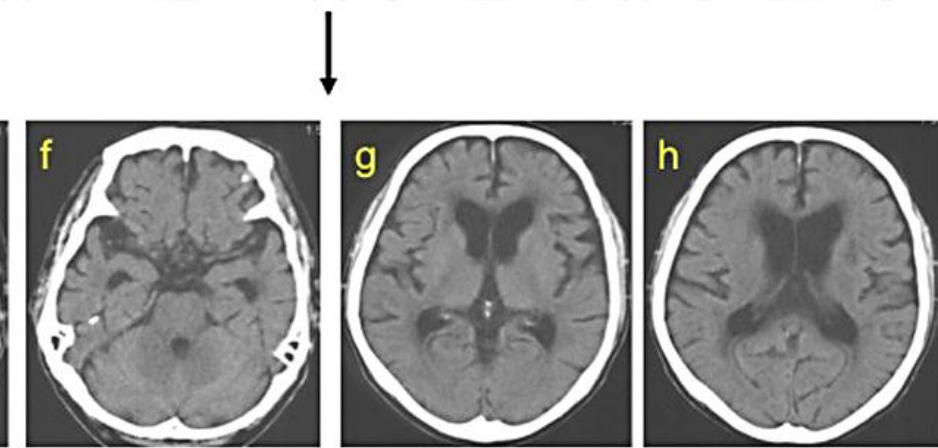

Post-RC therapy

Fig. 1. CT images of case 1. Multiple high-density areas surrounded by low-density areas (arrows) were observed prior to RC combination therapy (a-d). An effect was apparent after 6 cycles of RC combination therapy $(\mathbf{e}-\mathbf{h})$.
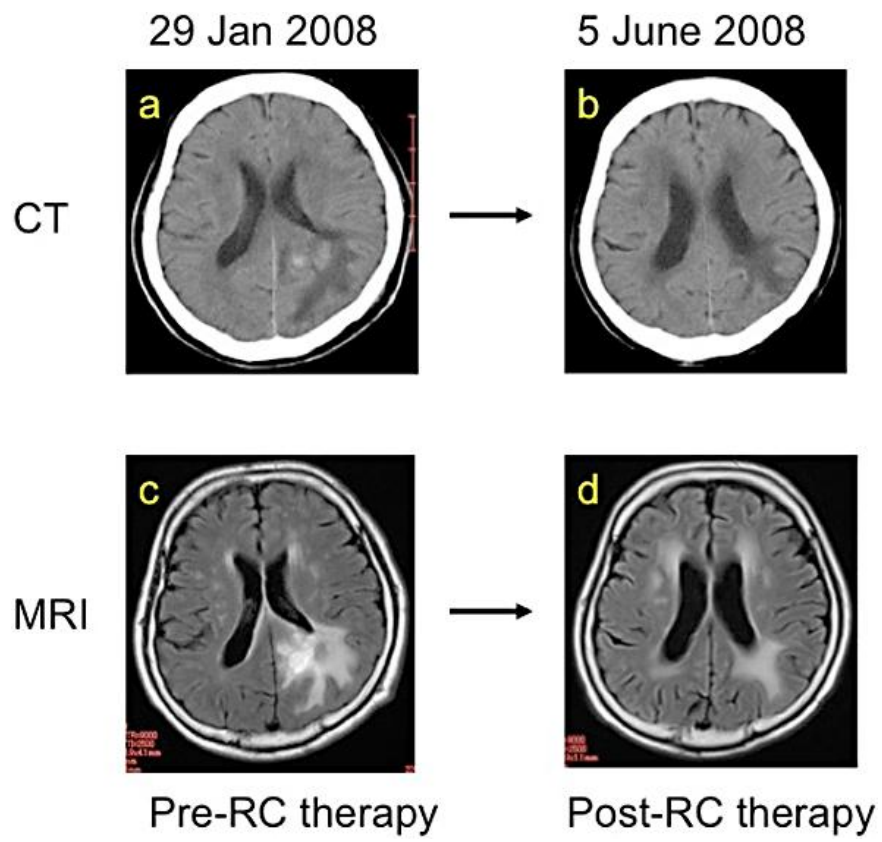

Fig. 2. CT (a, b) and MRI (c, d) images of case 2 . An effect was apparent (b, d) after 4 cycles of RC combination therapy. 


\section{References}

-1 Murray K, Kun L, Cox J: Primary malignant lymphoma of the central nervous system. Results of treatment of 11 cases and review of the literature. J Neurosurg 1986;65:600-607.

-2 Nelson DF, Martz KL, Bonner H, Nelson JS, Newall J, Kerman HD, Thomson JW, Murray KJ: Non-Hodgkin's lymphoma of the brain: can high dose, large volume radiation therapy improve survival? Report on a prospective trial by the Radiation Therapy Oncology Group (RTOG): RTOG 8315. Int J Radiat Oncol Biol Phys 1992;23:9-17.

-3 Loeffler JS, Ervin TJ, Mauch P, Skarrin A, Welnstein HJ, Canellos G, Cassady JR: Primary lymphomas of the central nervous system: patterns of failure and factors that influence survival. J Clin Oncol 1985;3:490494.

4 Merchut MP, Haberland C, Naheedy MH, Rubino FA: Long survival of primary cerebral lymphoma with progressive radiation necrosis. Neurology 1985;35:552-556.

$\checkmark 5$ Hiraga S, Arita N, Ohnishi T, Kohmura E, Yamamoto K, Oku Y, Taki T, Sato M, Aozasa K, Yosimine T: Rapid infusion of high-dose methotrexate resulting in enhanced penetration into cerebrospinal fluid and intensified tumor response in primary central nervous system lymphomas. J Neurosurg 1999;91:221230

-6 O’Brien P, Roos D, Pratt G, Liew K, Barton M, Poulsen M, Olver I, Trotter G: Phase II multicenter study of brief single-agent methotrexate followed by irradiation in primary CNS lymphoma. J Clin Oncol 2000;18:519-526.

-7 Rubenstein JL, Combs D, Rosenberg J, Levy A, McDermott M, Damon L, Ignoffo R, Aldape K, Shen A, Lee D, Grillo-Lopez A, Shuman MA: Rituximab therapy for CNS lymphomas: targeting the leptomeningeal compartment. Blood 2003;101:466-468.

>8 Delgado J, Canales MA, Garcia B, Alvarez-Ferreira J, Garcia-Grande A, Hernandez-Navarro F: Radiation therapy and combination of cladribine, cyclophosphamide, and prednisone as treatment of Bing-Neel syndrome: case report and review of the literature. Am J Hematol 2002;69:127-131.

-9 Fabianowska-Majewska K, Tybor K, Duley J, Simmonds A: The influence of 2-chloro-2'-deoxyadenosine on metabolism of deoxyadenosine in human primary CNS lymphoma. Biochem Pharmacol 1995;50:1379-1383.

10 Shah GD, Yahalom J, Correa DD, Lai RK, Raizer JJ, Schiff D, LaRocca R, Grant B, DeAngelis LM, Abrey LE: Combined Immunochemotherapy with reduced whole-brain radiotherapy for newly diagnosed primary CNS lymphoma. J Clin Oncol 2007;25:4730-4735. 